nor iritic adhesions to focal or ophthalmoscopical examination. Moreover, I think that assumption is quite out of court, for I have noticed it in others not syphilitic. As regards my second, a too liberal allowance of drops may be suggested. Putting aside the fact that the phenomena narrated soon supervened they had no larger quantity-and possibly less from the difficulties attendant-than older children who show no such symptoms. The atropine drops contained 1 per cent. or four grains to the ounce; the homatropine drops also contained 1 per cent. or four grains to the ounce. St. Ermin's Mansions, S.W.

\section{PNEUMONIA SUCCESSFULLY TREATED BY BRIN'S OXYGEN.}

By Robert Watering Bateman, M.R.C.S., L.S.A.

I SEND the following note for publication in THE LANCET, as I believe I am right in saying that this case is among the first treated by Brin's oxygen, as suggested and carried out by Dr. Lauder Brunton in the case of a clergyman in London. Mrs. W- aged sixty-three. I was called in whilst on my rounds on Jan. 31st of the present year to see this lady, and found her suffering from the prevailing epidemic (intluenza), with a temperature of $103^{\circ}$. After administering quinine the temperature came down, and all the influenza symptoms were subsiding until Feb. 6th, when pneumonia of the left base appeared, the temperature going up again to $102^{\circ}$ and the breathing to 36 . Consolidation appeared next at the right apex behind ; and, the patient becoming in a very dangerous state, I called Dr. Image of Bury St. Edmunds in consultation, and he advised Brin's oxygen. On administering the oxygen on the following day it was remarkable how the 'cyanosis disappeared after five minutes' inhalation. The inhalation was kept up for ten minutes the first time and given for a longer period subsequently. After that the oxygen was given twice daily, twenty minutes' inhalation a. a time, until MIarch 25th. The effect of the oxygen was very marked: after one administration, when at, her worst, the patient remarked that it made her feel quite hungry. It always removed the cyanosis, refreshed her, and kept her going: 'The apex of the right lung commenced to clear first and then the left base, until March 5th, when they were quite well. The administration of the oxygen was continued afterwards, as the convalescence was extremely slow, and it alwars did her good. There is one thing which ought to be well borne in mind-viz, that inflammations following epidemic influenza are of a very ataxic character and require free stimulation; for drugs, ether, ammonia and senega are the most useful, hyoscyamus when the cough is very troublesome. A good jacket poultice wrapped round the aching inflamed lung is the best local application, and it is best to put the water in the basin first and then add the linseed, stirring the while to make the poultice. The nurses I had from London last winter almost invariably made them in the opposite manner at first, making a Iumpy poultice; whilst the other way, putting the hot water in first and then adding the linseed, makes the poultice of an even consistence. There is another matter to be noticed in the pneumonia of influenza-viz., the erratic character of the inflammation. Commencing in one part-say, the base of one lung-it jumps, so to speak, right over to the other lung, and attacks perhaps the apex. There is no doubt that the influenza germ poisons the lungs, and it being erratic in its general behaviour may perhaps explain its attacking different portions of the lungs. In giving the gas it is best, when the valve has been turned on, to allow it to stream into the mouth direct, and not fill the bag which is in connexion with the tubes. It is a good thing to paint the chest with iodine liniment when the poultices are left off.

Wickhambrook, Newmarket.

\section{A CASE OF INFANTILE LACTATION}

By J. HILTON THOMPSON, M.D. VICT., LATE SENIOR RESIDENT MEDICAL OFFICER IN THE CHILDREN'S HOSPITAL, PENDLEBURY.

THE following case is of interest if only on account of its rarity. A healthy, well-devcloped male child, born at full term, slivwed abut funteen days after birth well-marked swelling of the mammary glands. The skin over the glands was congested; on pressure a thin, yellowish, milky fluid escaped drop by drop from the nipples. About two days after the condition was noticed a small, badly defined hardness could be felt in the left breast external to the nipple; moderate pressure caused no pain; the hardness remained for two days, gradually becoming less manifest. Lactation ceased in the right breast in five days and two days afterwards in the left. The general condition of the infant appeared to be unaffected.

Bolton, Lancashire.

\section{CASE OF SWALLOWING FISHBONES. \\ By J. R. White, M.B.}

ON March 8th last I was requested to attend Mr. D-_, and on arrival found him in bed complaining of some abdominal pain and also considerable thirst and retching; the temperature and pulse were, however, normal, but the tongue was coated. The history which he gave was that about a month previously he was partaking of fish in company with his wife when suddenly a somewhat large piece of fishbone slipped down his throat. He immediately rose from the table, and feeling choked, endeavoured to eject the offending substance, but without success, and it eventually passed into the stomach. Since the date of the occurrence he had not felt much inconvenience, but occasionally suffered from pain in the bowels and also found he could not exert himself or stoop much without causing pain. At last, as the pain got somewhat worse and sickness commenced, he sent for me The symptoms daily increased in severity. The pulse rose to 120 , also the temperature three or four degrees. The vomiting became constant and was accompanied by purging there were also pain and tenderness in the abdomen, especially in the ileo-cæcal region, and considerable perityphlitis ; the stools were of a typhoid character. The case was one in which the symptoms of gastro-enteritis and typhoid fever were closely combined. I had the advantage of the assistance of Dr. Frederick Carter in consultation In about a week several pieces of fishbone were passed per anum; the largest was a piece of the backbone about an incl long, with spines attached. Amelioration of the symptoms at once began, convalescence being established in a couplo of weeks. A sequel which I consider of interest in this case is that the patient was insured in an accident company and claimed compensation for the accident which he had met with, which he obtained.

Grays, Essex.

\section{PYROGALLTC ACID.}

By U. BanerJi, M.R.C.S., L.R.C.P. Lond.

ON May 15th last at about 3.30 P.M. two patients, husband and wife, took pyrogallic acid. From the statement of the husband and from the appearance of the bottle I consider that much more than a drachm of the acid was taken by each. The man told me that he swallowed two handfuls of the poison. On arriving at the house at 4 P.M. I found the male patient under the influence of liquor, and taking a bath under a water-tap. I administered an emetic to each, mustard and hot water. The male vomited, but the female did not. The vomit appeared to consist of water, mustard, mucus and a white matter looking like pyrogallic acid which I at the time held in my hand. Finding that the patient did not complain of anything, I simply ordered twenty drops of dilute nitro-muriatic acid to be taken every two hours, and at night six ounces of olive oil to be taken in three equal doses. Next morning (the 16th) I found the patients in their usual good health, but in both cases the tongue was stained deep black. The urine and stools passed over-night were quite normal. I did not order any medicine. On the 17th I took the following notes from the male patient (the female, I might hare mentioned, never complained of anything abnormal): "Sensation of drowsiness coming on at intervals; the patient likens it to that produced by opium. There is nausea, but no vomiting; slight paroxysmal numbness about the extremities and face; slight palpitation and dryness of the throat; tongue moist, still black; no abnormal sensation in the abdomen; passed a natural stool in the morning; heart sounds normal; pulse 62 in the minute; urine normal; perspiration rather scanty; 
no headache ; slept well." Since the above date the patients have been continuing in their usual good health. No one was present to see the acid actually swallowed, but the patients said that they took it in handfuls. The bottle which contained the acid was as large as a Howard's quinine phial two-thirds of its contents had disappeared. In the Medical Record (page 49) a case is recorded in which a patient suffering from psoriasis was poisoned by pyrogallic ointment applied to one half of his body, whilst to the other half chrysophanic acid ointment was applied for comparison. The difference can be explained only by the supposition that the acid acts differently when applied and when taken internally. Calcutta.

\section{A}

\section{HOSPITAL PRACTICE, BRITISH AND FOREIGN.}

Nulla autem est alia pro certo noscendi via, nisi quamplurimas et mor borum et dissectionum historias, tum aliorum tum proprias collecta habere, et inter se compare.-MORGAGNI De Sed. et Caus. Morb. habere, et inter

\section{ST. BARTHOLOMEW'S HOSPITAL.}

CASE OF HYSTERICAL PARALYSIS OF THE ABDUCTORS OF THE LARYNX AND OF THE DIAPHRAGM, ASSOCIATED WITH PECULIAR MOVEMENTS OF THE PALATE.

(Under the care of Dr. S. WEST.)

THE following is an interesting account of a patient suffering from paralysis of most important muscles due to hysteria. It is said, and with reason, that this clisease may simulate almost any other known disease; but there are usually certain conditions accompanying it which enable the physician or surgeon to recognise the origin of the trouble. Here "the very irregularity of the affection" enabled the physician to come to a correct diagnosis. Mad it not been for the careful examination of the larynx and the instructions based on the conclusion arrived at, it is possible that tracheotomy would have been performed for the urgency of the dyspnoea and a considerable element of danger introduced into the case as a result of the performance of the operation. The onset of bronchitis or of pneumonia might have proved fatal where the cliaphragm was already paralysed.

The patient, a woman aged thirty-eight, was admitted into the hospital on account of dyspncea. She stated that she had been in her usual health until Christmas time, when she was attacked with bronchitis, from which she had suffered once or twice before during the last three years. She had had a congh, but her breathing had not become difficult until a week before admission, in the middle of February. The patient was of medium height and well nourished. She was eridently suffering from dyspnœa, for the breathing was laboured and somewhat hurried, the alse nasi dilating, the face flushed, but without much cyanosis. The movements of the chest were peculiar, the breathing being entirely with the upper parts; the diaphragm was apparently paralysed, for it rid not descend at all on inspiration; and the abdomen, instead of being made prominent by inspiration, was retracted, as if the diaphragm was sucked up into the thorax, the lower intercostal spaces and the lower ribs being at the same time frawn in, as were also the supra-clavicular and epistemal spaces; the larynx remained fixed and showed no respiratory excursion. On auscultation nothing was to be heard out of the normal except a little wheezing. Inspiration was rather noisy, evidently owing to laryngeal stridor.

On examining the throat the movements of the palate were seen to be extreme, but of a peculiar kind, for on expiration it was raised with considerable violence as high as it could be, becoming on expiration quite flaccid, and falling down as if suddenly paralysed. The vocal cords, on laryngoscopic examination, were found to be in close approximation and separated by a chink not wider than half a line during inspiration. while on expiration they separated and assumed the cadaveric position. The cords themselves were quite white, though the patient spoke with a hoarse roice. As already stated, there was no respiratory excursion of the larynx at all, in spite of the laryngeal obstruction. The arytenoid cartilages did not move towards the mirldle line on inspiration, when the cords approximated, so that at that time a triangular chink was left in the posterior part of the glottis; on phonation, however, they moved freely towards each other and took their proper place. The condition of the larynx was clearly this, that the abductors were completely paralysed. Of the glottis closers the crico-arytenoidei laterales contracted on inspiration, possibly alone or in conjunction with the crico-arytenoidei to sone: extent. The arytenoidei postici and obliqui were not paralysed, for on phonation they contracted, bringins the arytenoid cartilages into approximation, thongh as the voice was hoarse it is probable that their contraction was not perfect, and the same was the case with the crico arytenoid. The action of the larynx was to a great extent perverted, as was that also of the palate, while the diaphragm was paralysed. The very irregularity of the affection suggested the correct diagnosis-viz., that of hysteria. This diagnosis was supported by the history obtained from the patient, that at the age of twenty-three she had suffered from a contraction of the throat and neck, so that she could not open her mouth, on account of which her front teeth were drawn and she was fed for some weeks by means of a stomach pump. The patient was now put under chloroform, but without any relief to the breathing. Perhaps she was not completely anæsthetised, for the breathing became unsatisfactory, and it did not seem prudent to push the chloroform further. Having formed the opinion that the affection was hysterical, Dr. West's instructions were given that the patient was on no account to be tracheotomised, but that if the dyspnœa became urgent intubation should be performed. This was done two days later, but without much success, for the tube was not long retained in position.

The interrupted current was then applied two or three times during the next two days externally to the larynx. The condition remained much the same until the $22 \mathrm{nd}$, when it was found that the arytenoid cartilages moved on inspiration about half way towards the middle line, thus increasing the dyspnœa somewhat. The breathing had from her admission been noticed to be much quieter during sleep. The patient was able to take solid food, but vomited at irregular times after taking it.

On the 26th, on examination in the morning, the condition was the same, but about mid-day it suddenly changed and the breathing became perfectly quiet. The diaphragm moved naturally, though not freely; the palate ceased to move at all on inspiration. The vocal cords occupied their normal position on quiet respiration and on deep inspiration separated widely as they ought to do; but it was observed that they were in constant oscillation, as if uncertain what exactly to do, sometimes being somewhat approximated and sometimes extremely retracted. On phonation the movements were natural, though approximation seemed to be harily complete, for the cords were not quite tense enough; and from this cause, as well as from a little laryngitis, the result of the intubation, the voice was still husky. The interrupted current had been used daily and the patient objected greatly to its application. On the 29 th the approximation of the cords had returned, though not quite to the same extent as at first and there was but little dyspnoea. On expiration, however, the cords did not recede more than a little and could not reach the cadaveric position by a long way. The same uncertainty about the contractions was evident, which was previously observed, so that on no two consecutive respirations were the positions exactly the same. The palate was stationary and the diaphragm moved now and then, but seemed to be equally uncertain what to do. This condition remained much the same whenever examination was made until March 2 nd, and then during the application of the battery to the abdomen the action of the diaphragm became normal, and on applying it to the larynx. and making the patient speak, the voice became quite clear, and both breathing and speaking remained natural till March 4th. On that morning the symptoms had returned, but an application of the battery caused their prompt disappearance, and at Dr. West's visit in the afternoon he found the diaphragm and palate acting naturally, but the action of the cords was peculiar, the uncertainty of their movements being greatly exaggerated-in fact, almost choreic-on quiet respiration, though deep inspiration steadied them for the time, as did phonation also. Phonation, however, appeared to be perfect, for the voice was clear and steady. 